\title{
BOUNDS FOR DIMENSIONS OF ODD ORDER NONSINGULAR IMMERSIONS OF $R P^{n}$
}

\author{
BY \\ HARUO SUZUKI
}

Introduction. Operations of exterior power in $\mathrm{KO}$-theory as well as StiefelWhitney classes are useful means to estimate the lowest dimension of an affine space in which real projective spaces and their product spaces are immersed or embedded (see, e.g., Milnor [1], Atiyah [2] and Suzuki [3]). The purposes of the present paper are to show, by similar means, that the operations in $K O$ theory as well as characteristic classes are applicable to higher order nonsingular immersions of real projective spaces, and to compare proofs and results of the former with those of the latter. In fact, proofs of the two methods are quite analogous.

Theorems (1.1), (1.4) are higher order nonimmersion theorems for differentiable manifolds. They are applied to real projective spaces in Theorems (1.2), (1.6) and we find bounds of dimensions of affine spaces in which real projective spaces are immersed without odd order singularities. The results (Theorem (1.1), (1.2)) are due to characteristic class arguments. Theorem (1.2) includes Feldman's examples for real projective planes [5. I] and we shall show more examples of the theorem. However, Corollary (1.5), Theorem (1.6) are derived from $\mathrm{KO}$ theory and they are useful for real projective spaces of some dimensions, where Theorem (1.2) does not work. In $\$ 2$, we explain symmetric tensor products of vector space bundle and introduce symmetric power operations in $\mathrm{KO}$-theory. These may be more than what we need below. We also explain, according to Pohl [4] and Feldman [5], the bundle of $p$ th order tangent vectors of the differentiable manifold and its relation to the immersion of the manifold in the affine space without singularities of order $p(p \geqq 1)$. Lemma (2.3) is stated in a proposition of Feldman [5. I] without proof. We shall add a brief proof of it.

In the last section, we prove all theorems.

1. Statements of results. Let $M$ be a $C^{r}$ differentiable manifold of dimension $n$, where $r \geqq p$ and $p$ is a positive integer. Let $f$ be a $C^{r}$ differentiable map of $M$ to an affine space $R^{N}$ of dimension $N$. We denote by $\tau(M)$ the tangent bundle of $M$ and denote by $\tau_{p}(M)$ the bundle of $p$ th order tangent vectors on $M$. The $p$ th order differential $\tau_{p}(f): \tau_{p}(M) \rightarrow \tau_{p}\left(R^{N}\right)$, followed by the iterated natural splitting

Received by the editors June 1, 1965. 
map $\nabla_{p}: \tau_{p}\left(R^{N}\right) \rightarrow \tau\left(R^{N}\right)$, is a homomorphism of vector space bundles covering $f$. Precisely, $\nabla_{p}$ is the composition $D_{1} D_{2} \cdots D_{p-1}$, where $D_{k}: \tau_{k+1}\left(R^{N}\right) \rightarrow \tau_{k}\left(R^{N}\right)$ is the natural $k$ th order symmetric linear connections on $R^{N}$. We set $v(n, p)=C_{n+p, p}-1$. If the map $\nabla_{p} \tau_{p}(f)$ is of maximal rank on each fiber, that is, $\operatorname{rank}\left(\left.\nabla_{p} \tau_{p}(f)\right|_{x}\right)=\max \{v(n, p), N\}, x \in M$, the map $f$ is said to $\mathrm{b}^{-}$affine nonsingular of order $p$. Let $W_{i}\left(\tau_{p}(M)\right)$ and $\bar{W}_{i}\left(\tau_{p}(M)\right)$ be the $i$ th Stiefel-Whitney class and the $i$ th dual Stiefel-Whitney class of $\tau_{p}(M)$.

THEOREM (1.1). Let $M$ be a differentiable $\left(C^{r}, r \geqq p\right)$ manifold of dimension $n$. Suppose that $M$ is immersed in $R^{v(n, p)+k}$ without affine singularities of order $p$.

(a) If $k \geqq 0$, then $\bar{W}_{i}\left(\tau_{p}(M)\right)=0$ for any integer $i>k$.

(b) If $k \leqq 0$, then $W_{i}\left(\tau_{p}(M)\right)=0$ for any integer $i>-k$.

We apply Theorem (1.1) to real projective spaces. We define integers $s(n, p)$ and $d(n, p)$ by

$$
\begin{aligned}
& s(n, p)=\max \left\{i \mid 0<i \leqq n,\left(\begin{array}{c}
C_{n+p, p}+i-1 \\
i
\end{array}\right) \neq \equiv 0 \bmod 2\right\} \\
& d(n, p)=\max \left\{i \mid 0<i \leqq n,\left(\begin{array}{c}
C_{n+p, p} \\
i
\end{array}\right) \neq 0 \bmod 2\right\}
\end{aligned}
$$

By the use of Lemma (2.2) which bears on computations of symmetric powers of tangent bundles and by the use of Lemma (2.3), we shall prove the following:

THEOREM (1.2). If $p$ is odd and if $-d(n, p)<k<s(n, p)$, then the real projective space $R P^{n}$ cannot be immersed in $R^{v(n, p)+k}$ without affine singularities of order $p$.

According to Feldman [5. I], there exist $p$ th order nonsingular immersions of $M$ into $R^{N}$ if $N \leqq v(n, p)-n$ or if $N \geqq v(n, p)+n$. Theorem (1.2) shows that in many cases of real projective spaces, $v(n, p)-n$ and $v(n, p)+n$ are best results. As a special case of Theorem (1.2), we obtain Feldman's examples [5. I, Theorem 6.1 (a) and (c)], that is, $R P^{2}$ cannot be immersed in $R^{v(2, p)+1}$ without affine singularities of order $p$ if $p=8 s+5$ or if $p=8 s+3$, and it cannot be immersed in $R^{v(2, p)-1}$ without affine singularities of order $p$ if $p=8 s+1$ or if $p=8 s+3$, for $s$ any non-negative integer. Besides them, we obtain easily:

Corollary (1.3) $\left({ }^{1}\right)$. If $p=2^{2 r+1} s+2^{2 r}-1, \quad 2^{2 r+1} s+2^{2 r}-3, \cdots$, $2^{2 r+1} s+2^{2 r}-2^{r}+3$, or $2^{2 r+1} s+2^{2 r}-2^{r}+1$ for $s$ any non-negative integer and if $-2^{r}<k<2^{r}$, then $R P^{2^{r}}$ cannot be immersed in $R^{v\left(2^{r}, p\right)+k}$ without affine singularities of order $p$.

(1) For instance, if $p=32 s+15$ or $325 s+13$ for $s$ any non-negative integer and if $-4<k<4$, then $R P^{4}$ cannot be immersed in $R^{v(4, p)+k}$ without affine singularity of order $p$. 
We use the operations $\gamma^{i}$ in $K O$-theory which are derived from exterior $i$ th powers of real vector space bundles. (See, e.g., Atiyah [2, p. 128].) We denote by $\tau_{p}^{0}(M)$ the element of $[K O]^{\sim}(M)$,

See $\S 3$ for details.

$$
v(n, p)-\tau_{p}(M) \text {. }
$$

THEOREM (1.4). Let $M$ be a compact connected differentiable $\left(C^{\boldsymbol{r}}, r \geqq p\right)$ manifold of dimension $n$. Suppose that $M$ is immersed in $R^{v(n, p)+k}$ without affine singularities of order $p$.

(a) If $k \geqq 0$, then $\gamma^{i}\left(\tau_{p}^{0}(M)\right)=0$ for any integers $i>k$.

(b) If $k \leqq 0$, then $\gamma^{i}\left(-\tau_{p}^{0}(M)\right)=0$ for any integers $i>-k$.

REMARK. We cannot apply Atiyah's method [2, p. 129] directly to prove (1.4) (a) for $i=k \geqq 0$.

Theorem (1.4) is regarded as a $p$ th order nonimmersion theorem and is stated as follows.

COROllaRY (1.5). Let $M$ be a compact connected differentiable manifold.

(a) If $\gamma^{i}\left(\tau_{p}^{0}(M)\right) \neq 0$ and $0 \leqq k<i$, then $M$ cannot be immersed in $R^{v(n, p)+k}$ without affine singularities of order $p$.

(b) If $\gamma^{i}\left(-\tau_{p}^{0}(M)\right) \neq 0$ and $-i<k \leqq 0$, then $M$ cannot be immersed in $R^{v(n, p)+k}$ without affine singularities of order $p$.

We apply Corollary (1.5) to real projective spaces. Let $\phi(n)$ be the number of integers $s$ such that $0<s \leqq n, s \equiv 0,1,2$ or $4 \bmod 8$. We define integers $\sigma(n, p)$ and $\delta(n, p)$ by

$$
\begin{aligned}
& \sigma(n, p)=\max \left\{i \mid i>0,2^{i-1}\left(\begin{array}{c}
C_{n+p, p}+i-1 \\
i
\end{array}\right) \neq \equiv 0 \bmod 2^{\phi(n)}\right\}, \\
& \delta(n, p)=\max \left\{i \mid i>0,2^{i-1}\left(\begin{array}{c}
C_{n+p, p} \\
i
\end{array}\right) \neq \equiv 0 \bmod 2^{\phi(n)}\right\} .
\end{aligned}
$$

By the use of Lemma (2.2), we prove:

THEOREM (1.6). If $-\delta(n, p)<k<\sigma(n, p)$, then the real projective space $R P^{n}$ cannot be immersed in $R^{v(n, p)+k}$ without affine singularities of order $p$.

As an example of Theorem (1.6), we consider third order nonsingular immersions of $R P^{2^{r-1}}$ where $W_{i}\left(\tau_{3}\left(R P^{2^{r}-1}\right)\right)(i>0)$ are trivial and Theorem (1.2) does not work. By direct calculations, we prove easily:

Corollary (1.7) $\left({ }^{2}\right)$. If $-\delta\left(2^{r}-1,3\right)<k<\sigma\left(2^{r}-1,3\right)$, then $R P^{2^{r-1}}$ cannot be immersed in $R^{C_{2}{ }^{r}+3,3}$ without affine singularities of order 3 .

(2) For instance, $R P^{15}$ cannot be immersed in $R^{N}$ without affine singularity of order 3 if $811<N<819$. 
Similar results are obtained for real projective spaces $R P^{n}$ if the factor of a power of 2 in $C_{n+p, p}$ is greater than $n\left({ }^{3}\right)$.

2. Symmetric $i$ th powers in $K O$-theory and affine nonsingular immersions of order $p$. Let $X$ be a finite connected CW-complex and $\mathscr{E}(X)$ be the set of isomorphism classes of real vector space bundles over $X . \mathscr{E}(X)$ is an Abelian semi-group with zero for the Whitney sum. The group $K O(X)$ is the minimal Abelian group which satisfies two conditions: (1) $K O(X)$ contains $\mathscr{E}(X)$. (2) The inclusion map $\theta: \mathscr{E}(X) \rightarrow K O(X)$ is a homomorphism.

By a method quite similar to exterior $i$ th power operations $\lambda^{i}$ (see, e.g., [2]) the symmetric $i$ th power operations will be introduced into $K O(X)$. Let $O^{i}$ be the operation of the symmetric $i$ th power in $\mathscr{E}(X)$, defined by the symmetrization of a tensor product of vector spaces. We identify a vector space bundle over $X$ with its isomorphism class in $\mathscr{E}(X)$. If $x$ is a vector space bundle of some dimension over $X$, then $O^{i} x, \mathrm{i}=0,1,2, \cdots$, are vector space bundles over $X$, i.e., $O^{i} x \in(X)$ and they have following formal properties:

$$
\begin{aligned}
& \text { (A) } O^{0} x=1, \\
& \text { (B) } O^{1} x=x \\
& \text { (C) } O^{i}(x \oplus y)=\sum_{j=0}^{i}\left(O^{j} x\right) \cdot\left(O^{i-j} y\right) \quad \text { for } x, y \in \mathscr{E}(X) .
\end{aligned}
$$

Now we prove:

Lemma (2.1). The operations $O^{i}, i=0,1,2, \cdots$, are uniquely extended over $K O(X)$ in such a way that they satisfy the condition (1).

Proof. We set

$$
\rho_{t}(x)=\sum_{i=0}^{\infty}\left(O^{i} x\right) t^{i},
$$

for an indeterminate $t$. By the properties (A), (C) of $O^{i}$, it determines a homomorphism of the additive semi-group $\mathscr{E}(X)$ to the multiplicative group $A(X)$ of formal power series in $t$ with coefficients in $K O(X)$ and with constant term 1. The homomorphism $\rho_{t}$ is extended over $K O(X)$ because it is the minimal Abelian group satisfying the conditions (1) and (2). Thus the operations $O^{i}$ are extended over $K O(X)$ and the extended operations are denoted by the same notations. They preserve properties (A), (B) and (C), and their uniqueness is obvious.

Let $M$ be a compact connected real differentiable $\left(C^{r}, r \geqq p\right)$ manifold. We prepare the following lemma which serves to compute $\tau_{p}^{0}\left(R P^{n}\right)$.

LeMma (2.2). We have

$$
\tau_{p}(M)=O^{p}(\tau(M)+1)-1 .
$$

(3) If we take $n=5, p=3$, this condition is satisfied. 
Proof. We consider the exact sequence of Pohl [4, Theorem 2.1],

$$
0 \rightarrow \tau_{p-1}(M) \rightarrow \tau_{p}(M) \rightarrow O^{p} \tau(M) \rightarrow 0 .
$$

Since all bundles in the sequence are real vector space bundles, the sequence splits. (See, e.g., Auslander and Mackenzie [6, p. 183].) By an induction on $p$, we obtain easily

$$
\tau_{p}(M)=\tau(M) \oplus O^{2} \tau(M) \oplus \cdots \oplus O^{p} \tau(M) .
$$

On the other hand, using properties (1) ((A), (B) and (C)) of symmetric $i$ th powers in $K O(M)$, it follows immediately that

$$
\begin{aligned}
O^{i}(\tau(M)+1) & =O^{i} \tau(M)+O^{i-1} \tau(M)+\cdots+\tau(M)+1 \\
& =\tau_{p}(M)+1
\end{aligned}
$$

Thus we obtain the formula of our lemma.

We consider relations between $\tau_{p}(M)$ and the immersion of $M$ in the affine space $R^{N}$ without affine singularities of order $p$ and prove the following results stated in [5. I].

Lemma (2.3)(Feldman). Suppose that $M$ can be immersed in $R^{N}$ without affine singularities of order $p$.

(a) If $N \geqq v(n, p)$, then there is an $(N-v(n, p))$-vector space bundle $\mu$ over $M$ such that

$$
\tau_{p}(M) \oplus \mu=N \text {. }
$$

(b) If $N \leqq v(n, p)$, then there is a $(v(n, p)-N)$-vector space bundle $\phi$ over $M$ such that

$$
\tau_{p}(M)=\phi \oplus N
$$

Proof. (a) Let $i: M \rightarrow R^{N}$ be the immersion of the assumption. By the definition of the mapaffine nonsingular of order $p$, the homomorphism $\nabla_{p} \tau_{p}(i)$ is a monomorphism of $\tau_{p}(M)$ into $\left.\tau\left(R^{N}\right)\right|_{M}$, the restriction of $\tau\left(R^{N}\right)$ over $M$, which is a trivial $N$-vector space bundle. Thus we can regard $\tau_{p}(M)$ as a subbundle of $\left.\tau\left(R^{N}\right)\right|_{M}$. We denote the quotient bundle $\left.\tau\left(R^{N}\right)\right|_{M} / \tau_{p}(M)$ by $\mu$. Obviously, $\mu$ is an $(N-v(n, p))$-vector space bundle over $M$. By the well-known splitting of the exact sequence of real vector space bundles,

$$
\left.0 \rightarrow \tau_{p}(M) \rightarrow \tau\left(R^{N}\right)\right|_{M} \rightarrow \mu \rightarrow 0,
$$

which we have used in the first part of the proof of Lemma (2.2), it follows that

$$
\tau_{p}(M) \oplus \mu=\left.\tau\left(R^{N}\right)\right|_{M} .
$$


(b) Let $i: M \rightarrow R^{N}$ be the immersion of assumption as (a). In this case, the homomorphism $\nabla_{p} \tau_{p}(i)$ is an epimorphism of $\tau_{p}(M)$ onto $\left.\tau\left(R^{N}\right)\right|_{M}$. Let $\phi$ be its kernel. By an argument similar to (a), the splitting of the exact sequence

$$
\left.0 \rightarrow \phi \rightarrow \tau_{p}(M) \rightarrow \tau\left(R^{N}\right)\right|_{M} \rightarrow 0
$$

leads the Whitney sum

$$
\tau_{p}(M)=\left.\tau\left(R^{N}\right)\right|_{M} \oplus \phi
$$

Thus Lemma (2.3) is proved. As for. Lemma (2.3) (a), see also Article 11 by Pohl stated in [7].

\section{Proofs of theorems.}

Proof of Theorem (1.1). (a) From the definition of the dual Stiefel-Whitney class and the relation (3) of Lemma (2.3) (a), it follows that

$$
\bar{W}_{i}\left(\tau_{p}(M)\right)=W_{i}(\mu)
$$

Since $\mu$ is the vector $k$-space bundle, we have $W_{i}(\mu)=0$ if $i>k$ and hence $W_{i}\left(\tau_{p}(M)\right)=0$ if $i>k$. (b) The proof is quite similar to that of (a). From the relation (4) of Lemma (2.3) (b), it follows that

$$
W_{i}\left(\tau_{p}(M)\right)=W_{i}(\phi) .
$$

Since $\phi$ is the vector $(-k)$-space bundle, we have $W_{i}(\phi)=0$ if $i>-k$ and hence $W_{i}\left(\tau_{p}(M)\right)=0$ if $i>-k$.

Proof of Theorem (1.2). By Lemma (2.2), we have

$$
\tau_{p}\left(R P^{n}\right)=O^{p}\left(\tau\left(R P^{n}\right)+1\right)-1
$$

Let $\xi$ be the Hopf bundle which is the line bundle associated to the natural covering $S^{n} \rightarrow R P^{n}$. It follows that

$$
\tau_{p}\left(R P^{n}\right)=C_{n+p, p} \xi^{p}-1 .
$$

If we denote by $W$ the total Stiefel-Whitney class $1+W_{1}+\cdots+W_{n}$ and denote by $\alpha$ the generator of the cohomology ring $H^{*}\left(R P^{n} ; Z_{2}\right)$, we obtain, for $p$ any odd,

$$
W\left(\tau_{p}\left(R P^{n}\right)\right)=(1+\alpha)^{C_{n+p, p}} .
$$

If we denote by $\bar{W}$ the total dual Stiefel-Whitney class $1+\bar{W}_{1}+\cdots+\bar{W}_{n}$, we obtain, for $p$ any odd,

$$
\bar{W}\left(\tau_{p}\left(R P^{n}\right)\right)=(1+\alpha)^{-C_{n+p, p}} .
$$

From Theorem (1.1) together with (5) and (6), Theorem (1.2) follows immediately. 
Now, we prove results obtained by operations in $\mathrm{KO}$-theory.

Proof of Theorem (1.4). (a) By Lemma (2.3) (a) for $N=v(n, p)+k, k \geqq 0$, we have

that is,

$$
\mu=\tau_{p}^{0}(M)+k
$$

$$
g \cdot \operatorname{dim} \tau_{p}^{0}(M) \leqq k,
$$

where $g \cdot \operatorname{dim} \tau_{p}^{0}(M)$ is the geometrical dimension of $\tau_{p}^{0}(M) \in[K O]^{\sim}(M)$. By Proposition (2.3) of Atiyah [2], we obtain $\gamma^{i}\left(\tau_{p}^{0}(M)\right)=0$ if $i>k$. (b) By Lemma (2.3) (b) for $N=v(n, p)+k, k \leqq 0$, we have

that is,

$$
\phi=-\tau_{p}^{0}(M)+(-k)
$$

$$
g \cdot \operatorname{dim}\left(-\tau_{p}^{0}(M)\right) \leqq-k .
$$

In an argument similar to (a), we obtain $\gamma^{i}\left(-\tau_{p}^{o}(M)\right)=0$ if $i>-k$.

Proof of Theorem (1.6.) If we set $x=\xi-1 \in[K O]^{\sim}\left(R P^{n}\right)$, from the first part of the proof of Theorem (1.2), it follows that for $p$ any odd,

$$
\tau_{p}^{0}\left(R P^{n}\right)=-C_{n+p, p} x
$$

By Atiyah [2], we obtain

and

$$
\gamma^{i}\left(\tau_{p}^{0}\left(R P^{n}\right)\right)=-2^{i-1}\left(\begin{array}{c}
C_{n+p, p}+i-1 \\
i
\end{array}\right) x
$$

$$
\gamma^{i}\left(-\tau_{p}^{0}\left(R P^{n}\right)\right)=(-2)^{i-1}\left(\begin{array}{c}
C_{n+p, p} \\
i
\end{array}\right) x .
$$

Therefore the result of Theorem (1.6) follows immediately from Corollary (1.5) together with (7) and (8).

\section{REFERENCES}

1. J. W. Milnor, Lectures on characteristic classes, Princeton, N. J., 1957 (mimeographed).

2. M. F. Atiyah, Immersions and embeddings of manifolds, Topology 1(1962), 125-132.

3. $\mathrm{H}$. Suzuki, Operations in KO-theory and products of real projective spaces, Mem. Fac. Sci. Kyushu Univ. Ser. A 18 (1964), 140-153.

4. W. F. Pohl, Differential geometry of higher order, Topology 1 (1962), 169-211.

5. E. A. Feldman, The geometry of immersions. I, II, Bull. Amer. Math. Soc. 69 (1963). 693-698; ibid. 70 (1964), 600-607.

6. L. Auslander and R. E. Mackenzie, Introduction to differentiable manifolds, McGrawHill, New York, 1963.

7. R. Lashof, Problems of differential and algebraic topology, Amer. Math. Soc. Summer Topology Institute, Seattle, Wash., 1963.

KYUSHU UNIVERSITY, FUKUOKA, JAPAN 